\title{
TARGETED STRATEGIES FOR TUBERCULOSIS IN AREAS OF HIGH HIV PREVALENCE: A SIMULATION STUDY
}

\author{
Georgina R. Mellor \\ School of Mathematics \\ University of Southampton \\ Highfield, Southampton, SO17 1BJ, U.K. \\ Elizabeth L. Corbett \\ London School of Hygiene and Tropical Medicine \\ Biomedical Research and Training Institute (at NIHR) \\ Josiah Tongogara Avenue (Cnr Mazoes) Avenues \\ Harare, PO Box CY1753, ZIMBABWE
}

\author{
Christine S.M. Currie \\ School of Mathematics \\ University of Southampton \\ Highfield, Southampton, SO17 1BJ, U.K. \\ Russell C.H. Cheng \\ School of Mathematics \\ University of Southampton \\ Highfield, Southampton, SO17 1BJ, U.K.
}

\begin{abstract}
We describe the analysis of a discrete event simulation model of tuberculosis (TB) and HIV disease, parameterized to describe the dual epidemics in Harare, Zimbabwe. The HIV epidemic in Sub-Saharan Africa is particularly severe and has led to a significant rise in TB cases. We use the model to evaluate new strategies for improved detection of TB cases in a high HIV prevalence setting. The structure of the model and its validation will be discussed, but the paper will focus on the analysis of the model output.
\end{abstract}

\section{INTRODUCTION}

Despite advances in treatment for tuberculosis (TB) (Dye et al 2005), the number of cases reported around the world continues to increase, with 8.9 million reported cases in 2004 (World Health Organization 2007). The greatest rate of increase in TB cases in recent years has been in the World Health Organization (WHO) Africa region, where the numbers exhibited an annual increase of $6 \%$ in 2000 . This increase can largely be attributed to HIV and approximately $31 \%$ of new TB cases in adults in the Africa region could be directly attributed to HIV (Corbett et al. 2003).

The change in TB epidemiology since the start of the HIV epidemic has led to a need to reassess the optimal control methods for TB disease. Worldwide, WHO has advocated the DOTS strategy (World Health Organization 2006a) since the early 1990s, but this has failed to keep pace with the TB epidemics of Sub Saharan Africa, which have been particularly affected by the HIV epidemic in the region (Dye et al 2005). There is therefore a need to reas- sess the policies developed in an era of low HIV prevalence to determine their utility in countries with high burdens of HIV infection.

In this paper we describe the results of a model designed to investigate the different modes of transmission of TB in a high HIV prevalence setting. In particular we investigate the effect of active case finding on the incidence rates and prevalence of TB disease in a community. Active case-finding involves targeted testing of the population for active TB disease, in contrast to the current policy of passive case finding where TB patients are only found when they present to the health services with symptoms indicative of TB disease. The model was described in an earlier paper (Hughes et al. 2006) with some preliminary results from the verification and validation process.

A brief description of the model is given in Section 2, including some changes that have been made since our earlier paper (Hughes et al. 2006). In Section 3 we detail the verification and validation processes that we have gone through and the results are presented in Section 4. We conclude in Section 5 with some suggestions for future research in this area.

\section{THE MODEL}

The basic model structure is similar to that described in an earlier paper (Hughes et al. 2006) but there have been some changes in the modeling of HIV and in the inclusion of risk factors, which are described below. The model describes the movement of individuals through eight possible stages of TB disease: susceptible to infection; 


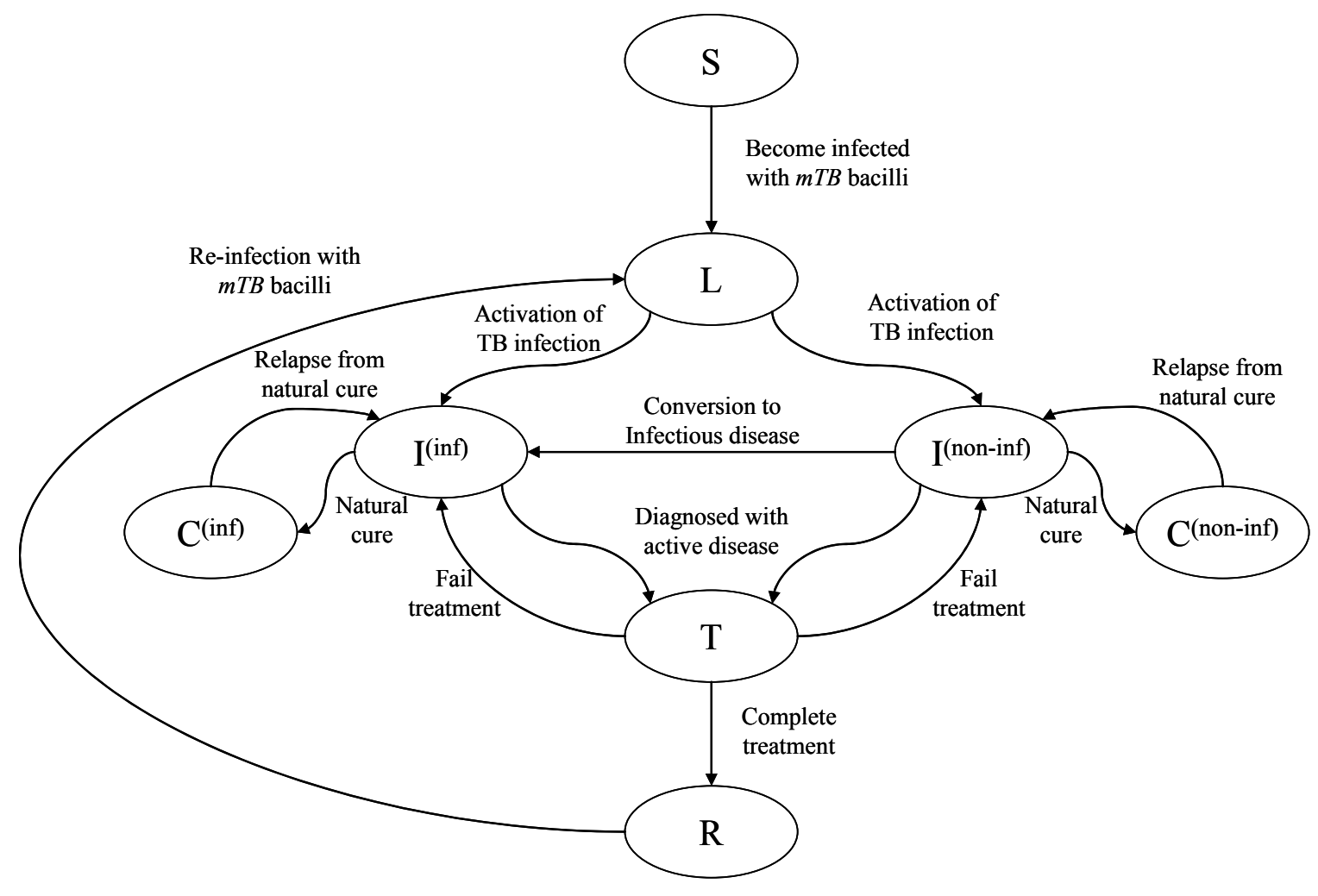

Figure 1: Schematic of progression through the TB disease states in the model, where $\mathrm{S}$ represents susceptible persons (uninfected with $m T B$ ); L those with an inactive $m T B$ infection; $\mathrm{I}^{\text {(inf) }}$ persons with active infectious TB disease; $\mathrm{I}^{\text {(non-inf) }}$ persons with active non-infectious TB disease, $C^{\text {(inf) }}$ persons who have spontaneously cured from active infectious TB disease; $C^{\text {(non-inf) }}$ persons who have spontaneously cured from active non-infectious TB disease; T persons undergoing treatment; $\mathrm{R}$ persons who have recovered from TB treatment.

latent infection; active infectious and non-infectious disease; naturally cured of infectious and non-infectious disease; undergoing treatment for TB disease and recovered from treatment for TB disease. The movements allowed between the disease states are shown in Figure 1.

\subsection{Transmission of TB}

The Mycobacterium tuberculosis (mTB) bacilli are usually transmitted by an infectious person coughing; therefore close contact is required for transmission, making transmission within the household particularly important. Therefore, we model transmission within the household separately from transmission within the general community. A further reason for separating household and community transmission is to allow us to evaluate strategies for active case-finding of persons with active TB disease. The current policy is to pursue passive case-finding for adults in endemic TB settings, i.e. waiting for sick people to present at the health services, which was made on the assumption that active case-finding for adults living in endemic TB settings is ineffective. This is based on research in low HIV preva- lence settings suggesting that transmission events between casual contacts greatly outnumber household transmission events, despite the closer contacts in the household (Rieder 2003). The impact of the HIV epidemic on the relative importance of household versus community transmission has not yet been fully assessed. As the duration of infectiousness of HIV-related TB is much briefer than that in HIVnegative patients, the HIV epidemic will have some effect on transmission (Corbett et al. 2004; Corbett et al. 2005; Currie et al. 2005) and may therefore impact on the optimal policy for active case-finding. We assume that each infectious TB patient will infect, on average, ten people per year (Currie et al. 2005), and we generate the number of household and random transmission events from Poisson distributions with means of 1 and 9 respectively.

\subsection{Risk Factors for TB Progression}

Patients can be HIV-negative or HIV-positive. HIVpositive individuals can be in the early, middle or late stages of the disease. Early stage or acute HIV refers to the first few months after a patient is infected with HIV. Dur- 


\section{Mellor, Currie, Corbett and Cheng}

ing this time the patient has transient but sometimes quite profound immunosuppression and we therefore assume that those with acute HIV have an increased susceptibility to developing active disease after initial infection with $m T B$. Because the window of immunosuppression is so brief, there is insufficient time for a previous latent infection to reactivate and therefore we assume that persons with acute HIV have no increased risk of reactivation. The middle stage of HIV refers to the time after recovery from acute HIV until a patient reaches the late stage (WHO stages 3 and above (World Health Organization 1990)). We assume that during the middle stage there is no increased risk of reactivation of an TB infection. Patients with late stage HIV are assumed to be more susceptible to activation of their $m T B$ infection and exhibit higher progression and death rates from TB (Morgan et al. 2002).

Infection with HIV is governed by a static model of HIV, which generates the number of HIV infections in each time step. The model relates available data on the prevalence of HIV infection to the incidence of HIV infection, a much more difficult quantity to measure. The age distribution of new HIV infections in a community is closely related to the age distribution of sexual activity. In order to accurately model the age dependence of HIV, we assign new HIV infections such that the proportion of new infections in an age group is proportional to the sexual activity of that age group, based on data from the UK (Johnson et al. 2001) and the proportion of the age group who are HIV-negative and therefore susceptible to infection. Thus, age groups which have a high proportion of HIVnegatives and in which the individuals have high levels of sexual activity will receive more of the new HIV infections than age groups with lower numbers of HIV-negatives and lower levels of sexual activity.

In addition to being a risk factor for HIV infection, a person's age will also affect their progression through the TB states. We incorporate age dependence using a similar approach to that used by Vynnycky and Fine (1997). Under these assumptions, children under ten years of age are unlikely to develop active disease when infected with $m T B$ bacilli; and as a person becomes older the risk of activation of a latent TB infection increases.

\section{MODEL VALIDATION}

Validating a simulation model involves ensuring that it is sufficiently accurate to answer the questions that it has been designed to address (Sargent 1998). The focus of our simulation study is an investigation of different modes of transmission of TB in a high HIV setting, leading to an evaluation of different strategies for active case finding for TB. Therefore, the most important aspects of the model are the transmission of TB and the effect of HIV on TB. In order to have credibility in the medical area, it is also impor- tant that the model is able to reproduce historical data of TB incidence and HIV prevalence.

\subsection{TB Incidence Model Output}

We have historical data for the prevalence of HIV infection among pregnant women attending antenatal clinics across Zimbabwe between 1984 and 1999 (World Health Organization 2006b), and the case notification rates per 100,000 members of the population for active TB disease between 1980 and 2002, also for Zimbabwe (World Health Organization 2007). An initial graphical comparison of the historical data with the model output of TB incidence, given in Figure 2, suggests that the model is able to reproduce TB incidence rates in the absence of HIV disease and shows a similar increase in TB cases following the start of the HIV epidemic. This suggests that the model provides a good description of HIV-negative TB and has captured most of the interactions between TB and HIV. However, the model does not produce as sustained an increase in TB cases as the historical data, perhaps suggesting that the model may be underestimating the effect of HIV on TB disease as the HIV epidemic matures.

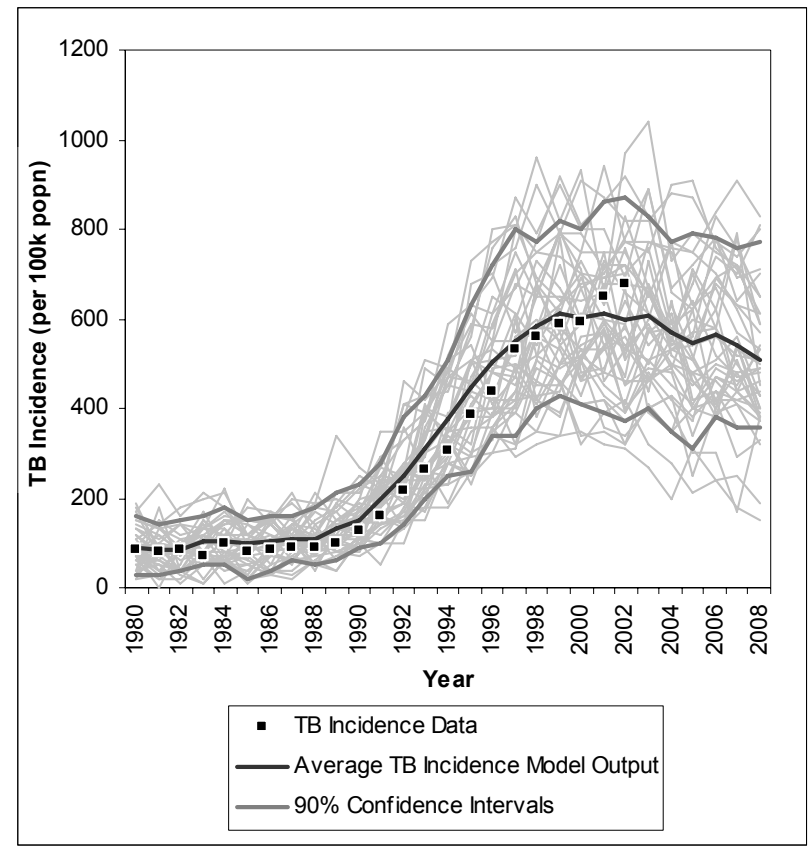

Figure 2: Model estimates of TB incidence in Zimbabwe. The graph shows the observed TB Incidence data from Zimbabwe and the output from 50 runs of the model, along with the average result and $90 \%$ confidence intervals.

\subsection{TB Transmission Dynamics}

Validating the modeling of TB transmission is more difficult as there are few data available. Most important to 


\section{Mellor, Currie, Corbett and Cheng}

our investigation is having the correct balance between transmission occurring within the household and that occurring within the wider community. Due to the lack of data available on the proportion of transmissions in these different settings, we used findings from an old study in India (Narain 1966) which investigated the distribution of persons infected with $m T B$ in households with and without confirmed cases. The study found that in children aged under 5 years, there was a prevalence of infection of $12 \%$ in households with bacteriologically confirmed cases as compared to $2 \%$ in households with no confirmed cases, giving an "infection intensity" (i.e. the ratio of percentage of contacts aged under 5 years infected among members of households without a case to the percentage of contacts in the same group infected among members of households with a case) (Hertzberg 1957) of 6 . The average output from 100 runs of the model suggested that the "infection intensity" within children under 5 years in our model population was 5.2. This implies that transmission dynamics are being correctly captured by the model however, having further data on the balance between household and random transmission would allow a more rigorous validation. As these data are unavailable, we will need to carry out a detailed sensitivity analysis on the parameter controlling the proportion of household versus random transmissions.

\subsection{HIV Model}

Other data exist that can be used for validating the model and increasing its credibility in the medical area. A particular aspect of the modeling that could be questioned is the use of a very simple HIV model. In order to justify this approach, we can compare the age-specific HIV prevalence of the model population with data from a similar setting. It has been observed (Williams et al. 2000) that the shape of the age-specific prevalence of HIV curves remains constant for different scales of HIV epidemic. It is possible to output from the model the proportion of HIV infections across the age groups and so compare the model output with the age-dependent HIV prevalence data observed by UNAIDS in South Africa (UNAIDS 2006) to see whether this pattern is observed. Figure 3 shows the distribution of HIV infection across the age groups for the model population in 2005 and the data for South Africa. Comparison with the model data shows that it is slightly overestimating the proportion of HIV infection amongst the 25-30 age group and underestimating amongst the 40+ age group. However, the overall distribution of HIV throughout the age groups is acceptable, especially when considering that the observations of Williams et al. (2000) came from data from 1998 when the HIV epidemic was still thriving. The authors acknowledged that, as the HIV epidemic saturates and starts to decline (as is the case with the data we are observing in 2005), the conclusions about a stable pattern of infection will be less valid, as the pattern of infection is likely to change.

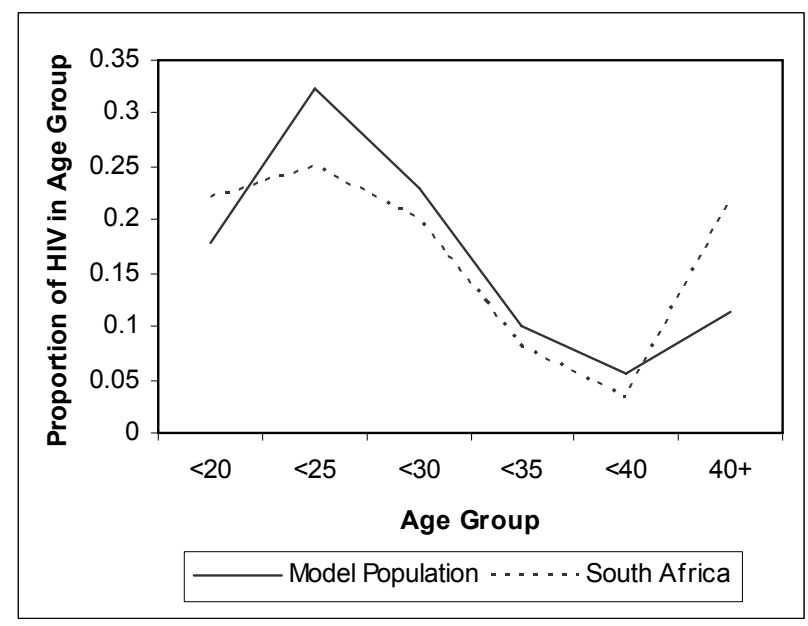

Figure 3: Comparison of the distribution of HIV throughout the age groups of the model's population and South Africa (Williams 2000).

\section{OUTPUT ANALYSIS}

We are interested in the impact of different strategies for active TB case-finding on the magnitude of the TB epidemic, where this is measured by the number of TB deaths and transmission events averted between the start of the interventions in 2008, and 2028. The strategies we consider are:

1. Investigating each member of a household in which there is a newly detected TB case or a death from TB, to see if there are any other undetected TB cases in the household

2. Investigating all members of the same number of households, where the households are selected at random

3. Investigating all members of the same number of households, where the households contain at least one HIV-positive person

4. Investigating each member of the household of all persons entering late-stage HIV to see if there are any other undetected TB cases in the household (we assume that it is around this time that an HIVpositive will approach the health services)

5. Investigating the same number of random households as in strategy 5

Strategies 1, 2 and 3 can be compared directly with each other as they require the same level of effort in terms of the number of households that need to be visited. Strategies 4 and 5 can be compared with each other and will pro- 
vide an insight into whether TB disease is clustered in HIV-positive households.

We run fifty iterations of the simulation model to compare the strategies, finding the difference between the total number of TB deaths and transmission events averted between the base case and the six strategies for each iteration. We minimize the variability by using the same start point in 2008 for each strategy in an iteration. This means that at the start of the trial period the simulation will have the same set of events scheduled and identical individuals with identical household structures for each strategy, with a start point being sampled at random in each iteration of the comparison. The results are presented in Figures 4, 5 and 6 .

Strategies 1,2 and 3 have a relatively small effect on the TB epidemic because of the small number of houses that are visited as part of this intervention. This is in line with conclusions from a study in Nepal which found contact investigations can be an inefficient activity (Wares et al. 2000) In this respect, strategies 4 and 5 perform much better, although as more than six times more households must be visited to complete these strategies, they would also be much more costly.

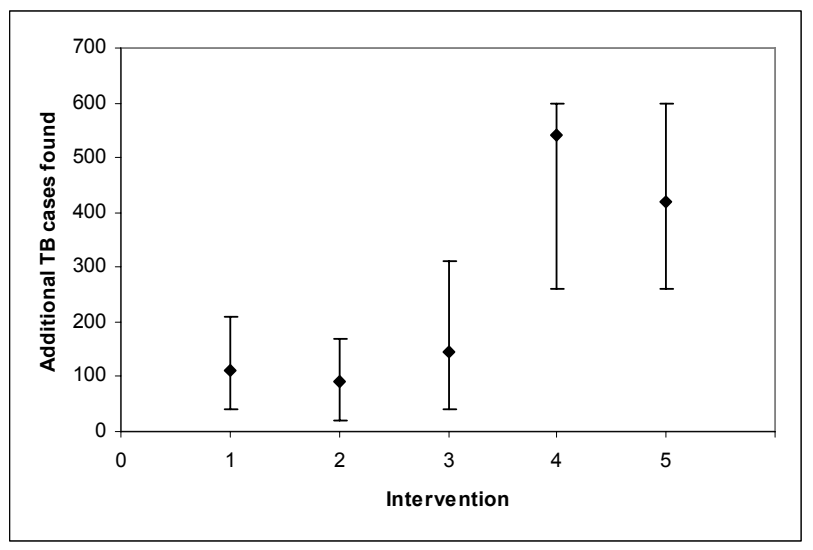

Figure 4: The number of additional TB cases found (per $100 \mathrm{k}$ population) by each of the household interventions when compared with the base case; $90 \%$ confidence intervals are included.

The relative benefits of employing strategy 1 (visiting households in which a person has been diagnosed with TB disease) versus strategies 2 (visiting the same number of random households) and 3 (visiting the same number of households with at least one HIV-positive) depend on the level of clustering of TB cases within households and how this is affected by the presence of an HIV-positive member. Analysis showed that strategy 3 was the most effective intervention for finding cases, suggesting that there is some clustering of TB cases and HIV infection. The probability of a household with a member about to commence TB treatment having a second person suffering from active TB disease is 0.009 , while for a household with at least one
HIV-positive member, the probability is 0.013 and for a general household the probability is 0.006 . This explains why strategy 3 appears to perform better than strategies 1 and 2, although the variability in the results is very high because of the small number of households being visited.

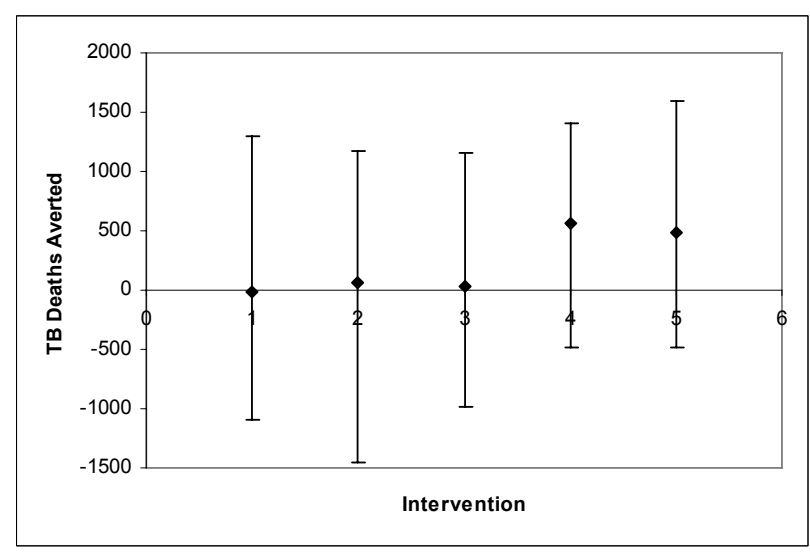

Figure 5: The number of TB deaths averted (per 100k population) by each of the interventions when compared with the base case; $90 \%$ confidence intervals are included.

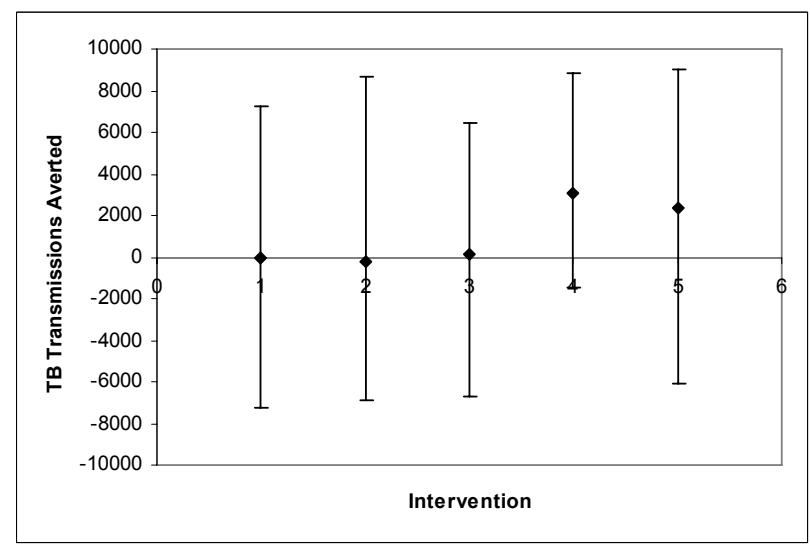

Figure 6: The number of TB transmissions averted (per $100 \mathrm{k}$ population) by each of the interventions when compared with the base case; $90 \%$ confidence intervals are included.

The relative benefit of employing strategy 4 over strategy 5 is clear. Visiting late-stage HIV households (households with a member with late-stage HIV) means that more cases are found and more TB deaths and transmissions averted compared to visiting households randomly. This can be explained by the clustering of HIV and TB infections that occurs within households. We are in the early stages of examining this clustering effect; however initial analysis has shown that households containing one or more TB cases are more likely to contain HIV individuals than households with no TB cases. This positive correlation be- 


\section{Mellor, Currie, Corbett and Cheng}

tween the number of HIV and TB infections within a household suggests that if TB is present in a household it is likely that HIV is too. It therefore follows that in order to find TB cases, it is more efficient to look in households with HIV infections than visiting households randomly.

\section{CONCLUSION}

The results of the modeling suggest that contact-tracing is relatively ineffective when the contacts are restricted to the household members of a person with active TB disease. This is partly due to the slow nature of TB disease, which means that any new infections within a household are unlikely to have developed into active disease by the time the first member of the household starts TB treatment. In addition, the strategy reaches only a small proportion of the population. We believe that active case-finding may appear a more attractive option if a wider range of contacts were found and so in future versions of the model we would like to include a more sophisticated social network. Finding the data to parameterize such a network may be difficult and this is the main limiting factor in this potential extension.

We also aim to incorporate TB preventive therapy into the model. Preventive therapy is given to a person with a latent $m T B$ infection to prevent it developing into active disease. We expect that adding preventive therapy to the above strategies will increase their efficiency, especially that of strategy 1 , in which household members of a person with active TB disease would be investigated for latent and active TB disease, administering TB treatment to any with active TB and preventive therapy to any with a latent $m T B$ infection.

When investigating the contacts of persons entering late-stage HIV, we find that it performs very well, averting the most TB deaths and TB transmission events per 100,000 population than any of the other active casefinding strategies. This is partly because there are more people being investigated in this intervention but it is also explained by the clustering of TB and HIV infections within households, such that the probability of finding a TB case within an HIV household is more than twice that of the general population.

\section{ACKNOWLEDGMENTS}

We thank the Engineering and Physical Sciences Research Council who helped fund this work as part of a $\mathrm{PhD}$ studentship for Georgina Mellor.

\section{REFERENCES}

Corbett EL, Bandason T, Dauya E, Makamure B, Cheung YB, Mtero-Munyati S, Matambo R, Godfrey-Faussett P, Hayes R, Churchyard GJ, Butterworth AE, Mason PR. 2005. HIV infection and the duration and control of prevalent tuberculosis disease in Harare, Zimbabwe. Proc. TSRU.: $28-39$.

Corbett EL, Charalambous S, Moloi VM, Fielding K, Grant AD, Dye C, De Cock KM, Hayes RJ, Williams BG, Churchyard GJ. 2004. Human immunodeficiency virus and the prevalence of undiagnosed tuberculosis in African gold miners. American Journal of Respiratory and Critical Care Medicine 170:673-679.

Corbett EL, Watt C, Walker N, Maher D, Williams BG, Raviglione MC, Dye C. 2003. The growing burden of tuberculosis: global trends and interactions with the HIV epidemic. Arch Intern Med 163: 1009-1021.

Currie CSM, Williams BG, Corbett EL. 2005. Assessing the Impact of HIV on the Annual Risk of TB Infection Using a Mathematical Model. Proc. TSRU.

Dye C, Watt CJ, Bleed DM, Mehran Hosseini S, Raviglione MC. 2005. Evolution of tuberculosis control and prospects for reducing tuberculosis incidence, prevalence and deaths globally. JAMA 293: 2767-2775.

Hertzberg G. 1957. The infectiousness of human tuberculosis; an epidemiological investigation. Acta Tuberc Scand Suppl 38:1-146.

Hughes GR, Currie CSM and Corbett EL. 2006. Modeling tuberculosis in areas of high HIV prevalence. In Proceedings of the 2006 Winter Simulation Conference, ed. L. F. Perrone, F. P. Wieland, J. Liu, B. G. Lawson, D. M. Nicol, and R. M. Fujimoto, 459-465. Piscataway, New Jersey: Institute of Electrical and Electronics Engineers, Inc.

Johnson AM, Mercer AH, Erens B, Copas AJ, McManus S, Wellings K, Fenton KA, Korovessis C, Macdowall W, Nanchahal K, Purdon S, Field J. 2001. Sexual behaviour in Britain: partnerships, practices, and HIV risk behaviours. Lancet 358: 1835-42.

Morgan D, Mahe C, Mayanja B, Whitworth JA. 2002. Progression to symptomatic disease in people infected with HIV-1 in rural Uganda: prospective cohort study. BMJ 324: 193-196.

Narain R, Nair S S, Rao G R, Chandrasekhar P. 1966. Distribution of tuberculosis infection and disease among households in a rural community. Bull World Health Organisation 34: 639-654.

Rieder HL. 2003. Contacts of tuberculosis patients in highincidence countries. International Journal of Tuberculosis and Lung Disease 7: S333-S336.

Sargent RG. 1998. Verification and validation of simulation models. In Proceedings of the 1998 Winter Simulation Conference, ed. D.J. Medeiros, E.F. Watson, J.S. Carson and M.S. Manivannan, 121-130. Piscataway, New Jersey: Institute of Electrical and Electronics Engineers, Inc.

Wares DF, Akhtar M, Singh S, Luitel H. 2000. Is TB contact screening relevant in a developing country setting? Experiences from eastern Nepal, 1996-1998. The In- 


\section{Mellor, Currie, Corbett and Cheng}

ternational Journal of Tuberculosis and Lung Disease. 4:920-924.

Williams BG, Gouws E, Colvin M, Sitas F, Ramjee G and Abdool Karim SS. 2000. Patterns of infection: using age prevalence data to understand the epidemic of HIV in South Africa. South African Journal of Science. 96: $1-9$.

World Health Organization. 1990. Acquired immunodeficiency syndrome (AIDS). Interim proposal for a WHO staging system for HIV infection and disease. Weekly Epidemiological Record 65: 221-224.

World Health Organization. 2006a. The Stop TB Strategy. <http://whqlibdoc.who. int/hq/2006/WH O_HTM_STB_2006.368_eng.pdf> [accessed Sēptember 2, $\overline{2} 007]$.

World Health Organization. 2006b. Epidemiological Factsheets on HIV/AIDS and Sexually Transmitted Infection, 2006 update. <http://www.who.int/ GlobalAtlas/predefinedReports/ EFS2006/EFS_PDFS/EFS2006_ZW.pdf> [accessed September 2, 2007].

World Health Organization. 2007. Global tuberculosis control: surveillance, planning, financing. <http: / / www.who.int/tb/publications / global_report/2007/pdf/full.pdf> [accessed September 2, 2007].

UNAIDS. 2006. AIDS epidemic update - Sub-Saharan Africa:

<http://data.unaids.org/pub/EpiRepor t/2006/2006 Epiupdate en.pdf> [accessed September 2, 2007].

Vynnycky E, Fine, PEM. 1997. The natural history of tuberculosis: the implications of age-dependent risks of disease and the role of reinfection. Epidemiology and Infection 119: 183-201.

\section{AUTHOR BIOGRAPHIES}

GEORGINA R MELLOR is a PhD student in the School of Mathematics at the University of Southampton. She previously obtained a BSc in Mathematics and an MSc in Operational Research from the University of Southampton. She is interested in the mathematical modeling of epidemics. Her email address is $<$ G.R.Melloresoton.ac.uk $>$ and her web page <wWW.maths. soton.ac.uk/ gm>.

CHRISTINE S. M. CURRIE is a lecturer of Operational Research in the School of Mathematics in the University of Southampton, where she also obtained her $\mathrm{PhD}$. In addition, she has an MPhys from Oxford University and an MSc in Operational Research from the University of Southampton. She is currently the Book Review editor for the Journal of Simulation and co-chair of the Simulation Special Interest Group in the UK Operational Research Society. Her re- search interests include mathematical modeling of epidemics, Bayesian statistics, revenue management, variance reduction methods and optimization of simulation models. Her e-mail address is <Christine.Curriedsoton.ac.uk> and her web page <wWW.soton.ac.uk/ ccurrie>.

ELIZABETH L. CORBETT MB BCh, $\mathrm{PhD}$, is a clinical epidemiologist and a Senior Lecturer in the Clinical Research Unit, London School of Hygiene and Tropical Medicine. She works full time in epidemiological research, based in the Biomedical Research and Training Institute, Harare, Zimbabwe. Her ongoing research projects are centred around the theme of TB epidemiology and control in high HIV prevalence areas, funded by the Wellcome Trust, UK. Her email address is <liz.corbettalshtm.ac.uk>.

RUSSELL C.H. CHENG is Professor of Operational Research in the School of Mathematics in the University of Southampton. He has an M.A. and the Diploma in Mathematical Statistics from Cambridge University, England. He obtained his Ph.D. from Bath University. He is a former Chairman of the U.K. Simulation Society, a Fellow of the Royal Statistical Society, Fellow of the Institute of Mathematics and Its Applications, Member of the Operational Research Society. His current research interests include: design and analysis of simulation experiments, variance reduction methods and parametric estimation methods and he has over seventy publications in these and related areas. He was a former Associate Editor of the ACM Transactions on Modeling and Computer Simulation and for Management Science and Editorial board member of the American Journal of Mathematical and Management Sciences. He is currently a technical board member of Simulation News Europe and is was a Joint Editor of the IMA Journal of Management Mathematics. His email address is <rchcemaths.soton.ac.uk> and his web page $<$ wWw maths. soton.ac.uk/staff/cheng $>$. 